\title{
Die Manifestation eines Nierenzellkarzinoms an der Nasenspitze
}

\author{
Manifestation of Renal Cell Cancer on the Nasal Tip
}

Autoren

Institute
D. K. Osmonov ${ }^{1}$, E. Jüttner ${ }^{2}$, I. Leuschner ${ }^{2}$, D. Melchior ${ }^{1}$, C. van der Horst ${ }^{1}$, K. P. Jünemann ${ }^{1}$

1 Klinik für Urologie und Kinderurologie, Universitätsklinikum Schleswig-Holstein, Campus Kiel

2 Institut für Pathologie im Universitätsklinikum Schleswig-Holstein, Campus Kiel

\section{Bibliografie}

DOI $10.1055 / \mathrm{s}-0029-1214901$

Online-Publikation: 2.7. 2009

Akt Dermatol 2009; 35:

441-443 @ Georg Thieme

Verlag KG Stuttgart · New York

ISSN 0340-2541

Korrespondenzadresse

Dr. med. D. K. Osmonov

Klinik für Urologie und

Kinderurologie

Universitätsklinikum Schleswig-

Holstein, Campus Kiel

Arnold-Heller-Straße 7

24105 Kiel

dosmonov@urology.uni-kiel.de

\section{Zusammenfassung \\ $\nabla$}

$25 \%$ bis zu 33\% aller Patienten mit Nierenzellkarzinom (NCC) haben bei der Erstdiagnose bereits Metastasen. Oft bleibt der Primarius lange nicht identifiziert und wird erst nach referenz-pathologischer Begutachtung per Zufall entdeckt. Wir berichten über eine Lokalisation des NCC an der Na-

\section{Einleitung}

\section{$\nabla$}

$25 \%$ bis $33 \%$ aller Patienten mit NCC haben bei der Erstdiagnose bereits Metastasen. Das NCC metastasiert in die Lunge 55\%, Lymphknoten 34\%, Leber $33 \%$, Skelett 32\%, Nebennieren 19\% und Haut $3-$ $5 \%$ [4]. Die Diagnose eines NCC wird häufig durch eine Vorsorge-Ultraschall-Untersuchung gestellt, dabei wird der Primarius in der Niere entdeckt. Die extrarenale Manifestation eines NCC wird oft nur zufällig diagnostiziert. Eine Literaturübersicht zeigt verschiedene NCC-Metastasen-Lokalisationen, die als Fallberichte beschrieben worden sind. Beschrieben sind Lokalisationen an der Haut, Bauchdecke, Thoraxwand [2,3], nasal und paranasal [9] sowie im Gastrointestinal-Trakt [6]. Es sind die orbitalen Metastasen [8], Harnblasenmetastasen [7] und Herzmetastasen [1] beschrieben.

\section{Kasuistik}

\section{Erst-|Aufnahmebefund}

Herr B. ist ein 68-jähriger Patient, der in Behandlung bei einem niedergelassenen Internisten aufgrund seiner koronaren 3-Gefäßerkrankung und COPD ist. Herr B. klagte unter anderem über Geschwürbildung an seiner Nasenspitze. Der Patient wurde in die Klinik für Dermatologie und Venerologie eingewiesen, wo nach Konsultation mit Mund-, Kiefer- und Gesichtschirurgen eine Indi- senspitze. Ein 68-jähriger Mann stellte sich beim Hausarzt mit einem ulzerierenden Tumor an der Nasespitze vor. Eine histologische Begutachtung ergab den Hinweis auf ein klarzelliges Karzinom. Eine CT-Staging-Untersuchung ergab den V.a. eine Neoplasie in der linken Niere. Es wurde eine Tumornephrektomie durchgeführt. Histologie: klarzelliges NCC pT1b, N0, M1, G2, R0, V0, L0.

kation zu Biopsie bzw. Tumorexzision gestellt wurde. Der Eingriff wurde unter Vollnarkose durchgeführt. Die histologisch-pathologische Begutachtung ergab den Hinweis auf eine Hautinfiltration durch einen ulzerierten, polypösen, hellzelligen Tumor, vereinbar mit einer Metastase eines hellzelligen NCC (Histologie 20565/2005), ( $\bullet$ Abb. 1). Eine referenz-pathologische immunhistochemische Untersuchung bestätigte die Diagnose eines klarzelligen Karzinoms (Histologie E 3969/06).

\section{Befunde diagnostischer Untersuchung}

Der Patient wurde in unserer onkologischen Sprechstunde vorgestellt. Wir führten eine Focussuche bzw. eine Staging-Untersuchung durch. Eine abdomen-sonografische Untersuchung ergab den Hinweis auf eine inhomogene, echoarme Raumforderung, max. Durchmesser 4,2 $\times 5,3 \mathrm{~cm}$, im Bereich des linken kranialen Nierenanteils, hochgradig verdächtig auf ein Malignom. Zur weiteren Evaluierung wurde eine Computertomografie des Thorax und Abdomens durchgeführt.

CT Abdomen in Spiraltechnik: Im Bereich des oberen Pols der linken Niere wurde eine knapp $5,6 \mathrm{~cm}$ in transversaler Ebene messende rundliche kontrastmittelaufnehmende Weichteilvermehrung gesehen. Es wurden keine pathologisch vergrößerten Lymphknoten festgestellt. 


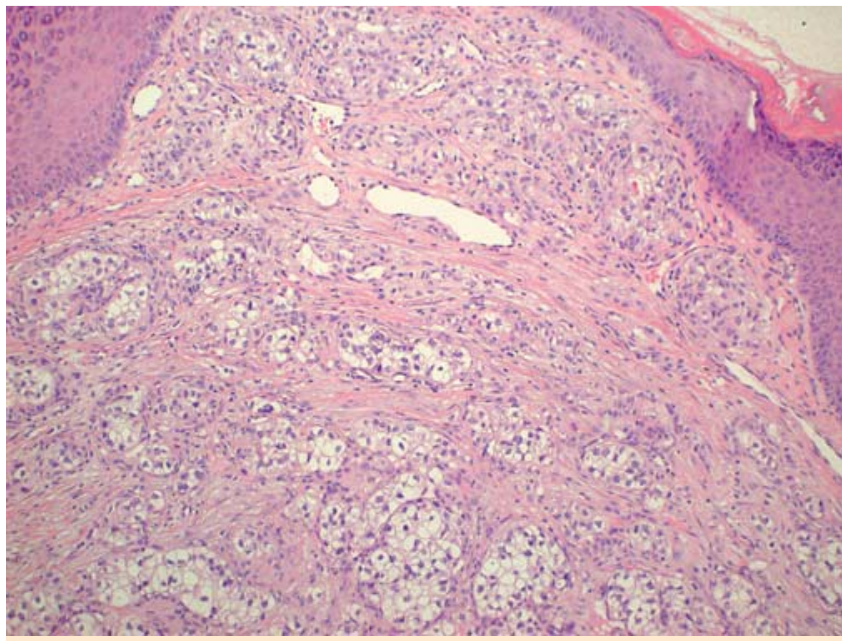

Abb. 1 Hautinfiltration durch einen ulzerierten, polypösen, hellzelligen Tumor, vereinbar mit einer Metastase eines hellzelligen NCC.

\section{Ergänzende Untersuchungen}

CT Thorax in Spiraltechnik: Vergrößerte mediastinale oder hiläre Lymphknoten lagen nicht vor. Im Segment 2 rechts fand sich ein ca. $1 \mathrm{~cm}$ großer Rundherd. Ein weiterer ca. $6 \mathrm{~mm}$ großer Rundherd wurde rechts an der Grenze zwischen Segment 3 und 6 diagnostiziert. Links im Segment 6 wurde fraglich ein weiterer kleinster Rundherd mit einem Durchmesser von ca. $3 \mathrm{~mm}$ entdeckt.

MRT des Hirns: Es wurde ein Verdacht auf intrazerebrale Filiae gestellt.

Knochenszintigrafie ergab den Verdacht auf eine disseminierte ossäre Filiarisierung.

\section{Therapie und Verlauf}

Zusammenfassend wurde ein Primärtumor in der linken Niere in der Größe von 5,6 cm, 2 metastasenverdächtige Lungenläsionen rechts, 1 metastasenverdächtige Lungenläsion links, disseminierte intrazerebrale und ossäre Läsionen festgestellt. Es besteht kein Zweifel, dass mehr als 80\% der Patienten mit Metastasen eines NCC innerhalb eines Jahres sterben. Die Indikation zur Tumornephrektomie bei Patienten im fortgeschrittenen Stadium sollte aktiv diskutiert werden und insbesondere bei vitalen Indikationen (wie z.B. Hb-relevante Hämaturie) gestellt werden. Herr B. hatte keine Symptomatik, biologisch war er altersentsprechend aktiv. Herr B. hatte einen ausgeprägten Therapiewunsch und war über mögliche OP-Risiken bzw. über möglicherweise keine Lebensverlängerung durch die Operation aufgeklärt. Letztendlich wurde die Indikation zur Tumornephrektomie durch ausgeprägten Patientenwunsch gestellt.

Die radikale Tumornephrektomie wurde in Seitenlage in typischer Art und Weise durchgeführt. Der postoperative Verlauf blieb komplikationslos. Die Histologie (E 4012/06) ergab den Hinweis auf einen stark eingebluteten, zum Teil nekrotischen $5,5 \times 4 \times 5 \mathrm{~cm}$ großen Tumor, aufgebaut aus sehr weiten zytoplasmatischen Tumorzellen. Es hatte sich kein invasives Wachstum in die Tumorkapsel gezeigt. Die Gefäßresektionsränder und der Ureterresektionsrand waren tumorfrei. Die entgültige Diagnose war: klarzelliges NCC. Nebennierenrindenhyperplasie. Tumorstadium: pT1b, Nx, M1, G2, R0, V0, L0.

Die Diagnose einer Hautmetastase an der Nasespitze, ausgehend von einem NCC, wurde damit bestätigt. Aufgrund der zerebralen Filiarisierung ist ein Ausschlusskriterium der immunmodulati- ven Chemotherapien nach Brinkmann und auch nach Phyrhönen gegeben. Nach einem ausführlichen Gespräch hat sich Herr B zu einer Therapie mit Multikinase-Inhibitoren entschieden. Diese wurde im Rahmen einer Studie durchgeführt.

\section{Diskussion}

$\nabla$

Die bis jetzt größte Studie beschreibt 306 Patienten mit NCC, wovon bei 10 Patienten $(3,3 \%)$ Hautmetastasen gefunden worden sind. 5 von diesen 10 Patienten hatten die Hautmetastasen schon vor der Nephrektomie und die weiteren 5 Patienten haben die Hautmetastasen nach einer Nephrektomie entwickelt. Das Follow up war 51 Monate [3]. 90\% der Patienten mit Hautmetastasen aus beiden Gruppen hatten bereits mindestens 1 weitere Metastase. Es sind Lungenmetastasen und Knochenmetastasen beschrieben. Die häufigste Lokalisation für die Hautmetastasen ist die Schädelhaut, weiterhin sind Hautlokalisationen an der Thorakalwand und an der Bauchdecke beschrieben. Alle Patienten mit Hautmetastasen hatten ein Tumorstadium T3b-T4 [3]. Andere Studien und Fallberichte kommen zu den gleichen Schlussfolgerungen und beschreiben die Hautmetastasen als schlechten prognostischen Faktor mit einer Lebenserwartung von 7 Monaten bis 23,8 Monaten [5,6,8] ( Tab. 1).

Der vorliegende Befund eines kleinen, mittelgradig differenzierten Primärtumors pT1b, Nx, M1, G2, R0, V0, L0 steht in Gegensatz zur Literatur und reflektiert in keiner Weise die Aggressivität des Tumors. Der Befund wurde in einer interdisziplinären urologisch-pathologischen Konferenz diskutiert. Es handelte sich eindeutig um ein NCC Tumorstadium pT1b, G2. Der Tumor war zum Teil nekrotisch verändert, sodass die Beurteilbarkeit, besonders die Malignitätsgradbestimmung, dadurch eingeschränkt war. Die Therapie mit Multikinase-Inhibitoren stellt einen neuen Therapiestandard dar. Langzeitbeobachtungen dieses Therapiekonzeptes sind sicherlich wünschenswert, werden aber möglicherweise angesichts der generell ungünstigen Prognose von Patienten mit multiplen Metastasen zu keinem zusätzlichen Erkenntnisgewinn führen. Die Prognose bei den Patienten mit Hautmetastasen eines NCC ist leider sehr schlecht.

\section{Zusammenfassung}

$\nabla$

Eine untypische Lokalisation eines NCC zeigt, wie mannigfach sich eine onkologische Erkrankung manifestieren kann. Bei einer urologischen Tumorfokussuche wird normalerweise auf die klassischen Symptome geachtet. Die Patienten mit Hautexanthemen werden durch Dermatologen, Mund-Kiefer-Chirurgen oder Otorhinolaryngologen untersucht. Es wird auf das mögliche NCC als Primarius nur differenzialdiagnostisch zum Schluss der Diagnostik untersucht. Oft bleibt der Primarius lange nicht identifiziert und wird erst nach referenz-pathologischem Begutachten per Zufall entdeckt. Die Sonografie der Nieren bei der Fokussuche eines Primarius in der Otorhinolaryngologie und Dermatologie ist unter anderen diagnostischen Verfahren technisch problemlos durchführbar und könnte im Zweifelsfall informativ sein.

Interessenkonflikt: Es besteht kein Interessenkonflikt. 
Tab. 1 Übersicht der bisher publizierten Fälle eines Nierenzellkarzinoms mit Hautmetastasen.

\begin{tabular}{|c|c|c|c|c|}
\hline Erstautor & Patienten (n) & Metastasenlokalisation & Diagnose & Überleben \\
\hline 1. Alghamdi A & 1 Patient männlich & Herz, Schulter, Bein & $\begin{array}{l}\text { präoperativ vor } \\
\text { Nephrektomie }\end{array}$ & Unter 1 Jahr \\
\hline 2. Barbagelata Lopez A & 1 Patient männlich & $\begin{array}{l}\text { Haut (untere linke } \\
\text { Extremität) }\end{array}$ & $\begin{array}{l}\text { postoperativ nach } \\
\text { Nephrektomie }\end{array}$ & Unter 1 Jahr \\
\hline 3. Dorairajan LN & $\begin{array}{l}10 \text { Patienten ( } 9 \text { männlich, } \\
1 \text { weiblich) mit Haut- } \\
\text { metastasen von } 306\end{array}$ & $\begin{array}{l}90 \% \text { der Patienten mit } \\
\text { Metastasen in Skalp und } \\
\text { Gesicht hatten zusätzlich } \\
\text { a. Lungen-, b. Knochen-Filiae }\end{array}$ & $\begin{array}{l}5 \text { präoperativ vor } \\
\text { Nephrektomie } \\
5 \text { postoperativ nach } \\
\text { Nephrektomie }\end{array}$ & 7 Monate \\
\hline 4. Koga S & 6 Patienten & $\begin{array}{l}\text { Kopf, Gesicht } \\
5 \text { von } 6 \text { Patienten hatten } \\
\text { zusätzliche Metastasen } \\
\text { a. Lungen, b. Leber, c. Knochen }\end{array}$ & $\begin{array}{l}1 \text { Patient präoperativ } \\
\text { vor Nephrektomie } \\
5 \text { Patienten postoperativ } \\
\text { nach Nephrektomie }\end{array}$ & 23,8 Monate \\
\hline 5. Loualidi A & 1 Patient & Duodenum & $\begin{array}{l}\text { postoperativ nach } \\
\text { Nephrektomie }\end{array}$ & 5 Jahre \\
\hline 6. Nakanishi Y & 1 Patient weiblich & $\begin{array}{l}\text { Harnblase, keine Fern- } \\
\text { metastasen }\end{array}$ & $\begin{array}{l}\text { präoperativ vor } \\
\text { Nephrektomie }\end{array}$ & Kurative Behandlung \\
\hline 7. Shome D & $\begin{array}{l}2 \text { Patienten männlich } \\
1 \text { Patientin weiblich }\end{array}$ & Auge, Orbita & $\begin{array}{l}2 \text { präoperativ } \\
1 \text { postoperativ }\end{array}$ & Patienten leben \\
\hline 8. Szymanski M & 1 Patient männlich & Kopf, Hals, Nasenhöhle & $\begin{array}{l}\text { präoperativ } \\
\text { vor Nephrektomie }\end{array}$ & 8 Monate \\
\hline
\end{tabular}

\section{Abstract}

\section{Manifestation of Renal Cell Cancer on the Nasal Tip \\ $\nabla$}

$25 \%$ to $33 \%$ of all patients with renal cell carcinoma (RCC) already have metastases when first diagnosed. The primarius is often not identified for a long time and discovered only accidentially after a reference pathologic assessment. We report on a localisation of the RCC on the nasal tip. A 68-year-old man visited his physician with an ulcerating tumor on the nasal tip. The histodiagnosis revealed a clear cell carcinoma. A CT-staging resulted in the suspicion of a neoplasia of the left kidney. A tumor nephrectomy was performed. Histodiagnosis: clear cell RCC pT1b, N0, M1, G2, R0, V0, LO.

\section{Literatur}

1 Huland H, Hautmann R. Nierentumore. In: Huland H, Hautmann R. Urologie. Heidelberg: Springer, 2006: 187-197

2 Barbagelata Lopez A, Ruibal Moldes M, Blanco Díez A et al. Cutaneous metastasis of a renal carcinoma: case report and review. Arch Esp Urol 2005; 58: $247-250$

3 Dorairajan LN, Hemal AK, Aron $M$ et al. Cutaneous metastases in renal cell carcinoma. Urol Int 1999; 63: 164-167

4 Szymański M, Szymańska A, Morshed K et al. Renal cell carcinoma metastases to nose and paranasal sinuses presenting as recurrent epistaxis. Wiad Lek 2004; 57: $94-96$

5 Loualidi A, Spooren PF, Grubben MJ et al. Duodenal metastasis: an uncommon cause of occult small intestinal bleeding. Neth J Med 2004; 62: $201-205$

6 Shome D, Honavar SG, Gupta P et al. Metastasis to the eye and orbit from renal cell carcinoma-a report of three cases and review in literature. Surv Ophthalmol 2007; 52: 213-223

7 Nakanishi Y, Arisawa C, Ando M. Solitary metastasis to the urinary bladder from renal cell carcinoma: a case report. Hinyokika Kiyo 2006; 52: 937-939

8 Alghamdi A, Tam J. Cardiac metastasis from a renal cell carcinoma. Can J Cardiol 2006; 22: 1231 - 1232

9 Koga S, Tsuda S, Nishikido M et al. Renal cell carcinoma metastatic to the skin. Anticancer Res 2000; 20: 1939-1940 